\title{
COVID-19 Pandemic and Male Fertility: Clinical Manifestations and Pathogenic Mechanisms
}

\author{
Adel Abdel-Moneim \\ Molecular Physiology Division, Faculty of Science, Beni-Suef University, 62511 Beni-Suef, Egypt \\ e-mail:adel_men2020@yahoo.com; adel.hassan@science.bsu.edu.eg \\ Received October 21, 2020 \\ Revised January 3, 2021 \\ Accepted January 7, 2021
}

\begin{abstract}
The novel coronavirus disease-2019 (COVID-19) pandemic, caused by severe acute respiratory syndrome coronavirus 2 (SARS-CoV-2), has been a major public health emergency worldwide with over 118.27-million confirmed COVID-19 cases and 2.62-million deaths recorded, as of March 12, 2021. Although this disease primarily targets lungs, damages in other organs, such as heart, kidney, liver, and testis, may occur. Testis is the cornerstone of male reproduction, while reproductive health is the most valuable resource for continuity of the human race. Given the unique nature of SARS$\mathrm{CoV}-2$, the mechanisms of its impact on the testes have yet to be fully explored. Notably, coronaviruses have been found to invade target cells through the angiotensin-converting enzyme 2 receptor, which can be found in the respiratory, gastrointestinal, cardiovascular, urinary tract, and reproductive organs, such as testes. Coronavirus studies have suggested that testes might be a potential target for SARS-CoV-2 infection. The first etiopathogenic concept proposed by current hypotheses indicates that the virus can invade testes through the angiotensin-converting enzyme 2 receptor. Next, the activated inflammatory response in the testes, disease-associated fever, and COVID-19 medications might be implicated in testicular alterations. Although evidence regarding the presence of SARS-CoV-2 mRNA in semen remains controversial, this emphasizes the need for researchers to pay closer attention to sexually transmitted diseases and male fertility after recovering from COVID-19. In this review the latest updates regarding COVID-19-associated testicular dysfunction are summarized and possible pathogenic mechanisms are discussed.
\end{abstract}

DOI: $10.1134 / \mathrm{S} 0006297921040015$

Keywords: COVID-19, SARS-CoV-2, testis, manifestations, pathogenicity, male fertility

\section{INTRODUCTION}

The novel coronavirus disease-2019 (COVID-19) pandemic, caused by the severe acute respiratory syndrome coronavirus 2 (SARS-CoV-2), is the current global public health issue with exponential rise in infections worldwide [1]. COVID-19 pandemic has affected 213 countries with more than 118.27-million confirmed cases and 2.62-million disease-associated deaths as of March 12, 2021 [2]. Recent reports indicate that almost $58 \%$ of those infected with SARS-CoV2 are male, making

Abbreviations: ACE2, angiotensin-converting enzyme 2; CD3, cluster of differentiation 3; COVID-19, the novel coronavirus disease-2019; FSH, follicle-stimulating hormone; HIV, human immunodeficiency virus; IL, interleukin; $\mathrm{LH}$, luteinizing hormone; SARS-CoV, severe acute respiratory syndrome coronavirus; SARS-CoV-2, severe acute respiratory syndrome coronavirus 2; T, testosterone; TMPRSS2, transmembrane protease serine 2 . the male sex as one of the risk factors for COVID-19 [3]. Although COVID-19 primarily manifests as an acute respiratory illness, it can also affect other organs such as the kidney, heart, testes, and liver [4].

Previously testicular damage and spermatogenesis abnormalities were observed in patients infected with SARS: all SARS-CoV-infected testes displayed widespread germ cell destruction, few or no spermatozoon in the seminiferous tubules, and leukocyte infiltration [5]. Thus, due to the similarities between SARS-CoV and SARS-CoV-2, the orchitis-like syndrome in male patients with SARS-CoV-2 has been suspected [6]. The main host receptor of SARS-CoV or SARS-CoV2 is angiotensin-converting enzyme 2 (ACE2) receptor. The ACE2 receptors are widespread and highly expressed in the heart, lungs, kidneys, and testes [7]. This suggests that SARS-CoV-2 could likely exert adverse effects on reproductive system in males through ACE2 or other factors, leading to testicular damage in male patients. Notably, 
more attention should be given to the potential risk of SARS-CoV-2 infection on the male fertility [8]. Testes are the cornerstone of male reproduction, while reproductive health is the most valuable resource for continuity of the human race. However, little is known about the potential short- and long-term impact of COVID-19 on the male reproductive system. This review provides insights into clinical manifestations and possible pathogenicity of the SARS-CoV-2 infection in relation to testicular injury based on the latest studies.

\section{COVID-19 AND CLINICAL MANIFESTATIONS RELATED TO TESTICULAR INJURY}

Testis orchitis. Previous studies have reported that a number of viruses could infect testes, including mumps virus, human immunodeficiency virus (HIV), and SARS$\mathrm{CoV}[5,9]$. Regarding the novel SARS-CoV-2, which shares $76 \%$ amino acid sequence homology with the SARS-CoV, one can hypothesize that SARS-CoV-2 may have the ability to invade testes. Indeed, Gagliardi et al. reported the case of orchi-epididymitis associated with SARS-CoV-2 infection [10]. Moreover, six patients with SARS-CoV showed signs of orchitis and testicular injury including reduced numbers of germ cells and apoptotic death with interstitial leukocyte infiltration. Additionally, histopathological findings showed inflammatory infiltrations and accumulation of immunoglobulin-G (IgG), particularly in seminiferous epithelium, interstitium, degenerated germ cells, and Sertoli cells [5]. This finding is consistent with the results presented in the study of Pan et al., where 6 out of 34 men who recovered from SARS-CoV-2 infection developed signs of scrotal discomfort [11]. Additionally, the study of serious COVID19 cases in Brazil identified fibrin microthrombi orchitis in two of the investigated testes [12]. Similarly, postmortem examination of 12 COVID-19 male patients in China showed marked seminiferous cellular damage, reduced Leydig cell number, and moderate lymphocytic inflammation [13]. One study suggested that the orchitis may have resulted from vasculitis considering correlation between the COVID-19 and coagulation disorders, and that the segmental vascularization of the testes could cause an orchitis-like syndrome [6]. The aforementioned findings could suggest that SARS-CoV-2 infection may contribute to testicular ultrastructural lesions and orchitis in the severely infected males.

Sex hormone abnormalities. Regarding androgen secretion, the decreased total testosterone and calculated free testosterone with elevated luteinizing hormone (LH) levels recorded in the severe cases of COVID-19, have been significantly correlated with the increased plasma lactate dehydrogenase and ferritin levels and as well as with higher neutrophil counts and reduced lymphocyte counts [14]. These results are in agreement with the findings of another study that showed substantial reduction in the serum testosterone levels in 113 patients $(51.1 \%)$ with severe COVID-19, suggesting this parameter as a negative predictor of COVID-19 progression [15]. In addition, the recent study on 119 men with COVID-19 observed that the infected men had slightly lower overall serum testosterone levels, markedly higher serum LH, and lower testosterone: $\mathrm{LH}$ and follicle-stimulating hormone (FSH): $\mathrm{LH}$ ratios compared to 273 age-matched healthy men [16]. Similarly, the German study revealed that the critically ill male COVID-19 patients exhibited elevated LH and FSH levels with decreased testosterone and dihydrotestosterone levels [17]. Interestingly, the serum LH elevation in male patients with COVID-19 could possibly inhibit the hypothalamic-hypophyseal-testicular axis and perhaps explain the primary Leydig cell injury [18]. Moreover, low testosterone level had been the most important predictive hormonal factor for in-hospital mortality in the group of aged male patients [19]. Accordingly, COVID-19 may induce acute male hypogonadism that is clinically manifested as a reduction in testosterone levels (table).

Virus detection in seminal fluid. To date, no records on the sexual transmission of SARS-CoV-2 exist, while evidence regarding the presence of SARS-CoV-2 in semen remains limited. Recently, the contradictory findings have been reported regarding the presence of SARS-CoV-2 in the semen of patients diagnosed with COVID-19. Meanwhile, individuals infected with SARS-CoV-2 should take all possible precautions to minimize the possible risk of transmitting the infection via sexual intercourse [20]. Although actual fertility situation in the patients diagnosed with COVID-19 is still to be explored, the American Society for Reproductive Medicine (ASRM) and the Society for Assisted Reproductive Technology (SART) had already published warnings regarding the SARS-CoV-2 transmission via sexual intercourse [21]. Importantly, among the 38 patients with COVID-19 who provided semen specimens, 6 patients $(15.8 \%)$ had positive results for SARS-CoV-2 RNA, including 4 of 15 patients $(26.7 \%)$ who were at the acute stage of infection and 2 of 23 patients (8.7\%) who were recovering [22].

In contrast, Pan et al. reported no virus in patients' semen 29-36 days after recovery, although viral orchitis symptoms were observed in $19 \%$ of the patients with COVID-19 and six men displayed mild scrotal discomfort at the time of disease [11]. Furthermore, Song et al. [23] collected 12 semen samples from the COVID-19 patients and one postmortem testicular biopsy. SARS-CoV-2 RNA was not detected in the semen samples and testicular biopsy. The authors concluded, based on the fact that SARS-CoV-2 was not identified in the semen and testis samples, that the probability of testicular infection during the early and symptomatic phase of the infection is low but cannot be ruled out, when certain organs such as lung, heart, and gut are infected. In line with this, Holtmann et al. [24] found no SARS-CoV-2 RNA in the 
Testicular dysfunction manifestations and pathogenicity associated with SARS-CoV-2 infection

\begin{tabular}{|c|c|c|c|}
\hline & Testicular dysfunction & Number & References \\
\hline $\begin{array}{l}\text { Clinical } \\
\text { manifestations }\end{array}$ & $\begin{array}{l}\text { viral orchitis recorded in } 6 \text { patients; no virus detected in the semen } \\
\text { seminiferous cellular damage was observed in the specimens from } 12 \text { patients died from } \\
\text { COVID-19; no virus detected in testes in } 11 \text { deceased patients, but } 1 \text { was positive } \\
\text { decreased levels of total and free testosterone; increased serum level of LH was detected } \\
\text { significant reduction in the serum testosterone level in } 113 \text { patients ( } 51.1 \%) \\
\text { with severe COVID-19 } \\
\text { moderate decrease in the testosterone levels; LH level was higher; testosterone:LH } \\
\text { level and FSH:LH ratio were lower } \\
\text { LH and FSH levels were elevated; both testosterone and dihydrotestosterone levels } \\
\text { decreased } \\
\text { SARS-CoV-2 RNA was not detected in } 12 \text { semen samples and testicular biopsy } \\
\text { six patients ( } 15.8 \% \text { ) had positive SARS-CoV-2 } \\
\text { no virus was detected in } 34 \text { semen samples }\end{array}$ & $\begin{array}{l}34 \\
12 \\
31 \\
221 \\
119 \\
35 \\
12 \\
38 \\
34\end{array}$ & $\begin{array}{l}{[11]} \\
{[13]} \\
{[14]} \\
{[15]} \\
{[16]} \\
{[17]} \\
{[23]} \\
{[22]} \\
{[24]}\end{array}$ \\
\hline $\begin{array}{l}\text { Infection via } \\
\text { ACE2 receptor }\end{array}$ & $\begin{array}{l}\text { ACE2 and TMPRSS } 2 \text { expression detected mainly in seminiferous duct cells, sper- } \\
\text { matogonia, Leydig cells, primordial germ cells, and Sertoli cells }\end{array}$ & & {$[7,27,28-33]$} \\
\hline $\begin{array}{l}\text { Inflammatory } \\
\text { response and } \\
\text { persistent fever }\end{array}$ & $\begin{array}{l}\text { cytokines could cause orchitis in patients by inducing inflammatory response } \\
\text { hyper-inflammatory conditions with persistent fever affect testis function }\end{array}$ & & $\begin{array}{c}{[5,33,36,37]} \\
{[39-43]}\end{array}$ \\
\hline $\begin{array}{l}\text { Drug-related } \\
\text { testicular injury }\end{array}$ & $\begin{array}{l}\text { glucocorticoids and stress cause testes injury; ribavirin reduced testosterone levels } \\
\text { and inhibited spermatogenesis } \\
\text { lopinavir/ritonavir could inhibit spermatogenesis } \\
\text { chloroquine phosphate affects spermatogenesis and epididymal function }\end{array}$ & & $\begin{array}{c}{[22,45]} \\
{[46]} \\
{[47]}\end{array}$ \\
\hline
\end{tabular}

Note. Designations: ACE2, angiotensin-converting enzyme 2; FSH, follicle-stimulating hormone; LH, luteinizing hormone; SARS-CoV, severe acute respiratory syndrome coronavirus; TMPRSS2, transmembrane protease serine 2 .

semen of 34 recovered or acutely infected males with SARS-CoV-2 43 days after diagnosis on average. Furthermore, among the 12 deceased patients with COVID-19, 11 were negative for SARS-CoV-2 RNA in the testicular tissue, while one was positive for the virus. However, the patients with COVID-19 had been found to display seriously damaged seminiferous tubules, decreased Sertoli cells, and mild inflammatory infiltrates in the interstitium [13] (table). Therefore, evidence available from 132 cumulative patients over 7 studies revealed that the virus was present in the semen of $7(5 \%)$ patients with COVID-19 [11, 13, 22-26].

Moreover, Ma et al. [16] reported that 4 patients with COVID-19 (33.3\%, 4/12) had low sperm motility. Duration between the semen collection and the disease onset ranged from 56 days to 109 days (with a median of 78.5 days). However, a marked negative effect on the semen parameters such as sperm concentration, total sperm count, and the total number of progressive motility was observed in the recovered participants with mild symptoms and need for hospitalization compared to the control group. This finding indicates that SARS-CoV-2 infection has short-term effects on spermatogenesis in patients with mild symptoms associated with COVID-19 [24]. Notably, Yang et al. [13] stated that COVID-19 can induce testicular tissue injury and affect fertility, particularly in young men, which highlights the need for examining testicular function after recovery.

\section{COVID-19 AND TESTICULAR PATHOGENICITY}

Direct testicular invasion of SARS-CoV-2. ACE2 receptors, which are omnipresent and highly expressed in 
several organs such as heart, gut, lungs, kidneys, testes, and brain [7], directly influence invasion of the three coronavirus strains into human cells: SARS-CoV, NL63, and SARS-CoV-2. Seminiferous tubules represent up to $90 \%$ of the human testis tissues, the Sertoli cells and the germ cells express ACE2 making them a potential site for SARS-CoV-2 infection that subsequently impacts spermatogenesis (figure). Interestingly, testicular cells showed the highest level of ACE2 mRNA in the seminiferous duct cells, spermatogonia, Leydig cells, primordial germ cells, and Sertoli cells. Considerable seminiferous tubular damage, decreased number of Leydig cells, and moderate lymphocyte inflammation was reported in the studies of patients with COVID-19 [7, 27]. The physiological roles of ACE2 in Leydig cells include control of steroidogenesis and spermatogenesis, modulation of testosterone synthesis, and regulation of the local vascular regulatory system to balance the volume of interstitial fluid by controlling conversion of Angiotensin II to Angiotensin I $[14,28]$. Additionally, histopathological studies of biopsies from COVID-19 male patients showed impaired spermatogenesis in three out of six samples. Also, histological staining of one sample from the patient with COVID-19 demonstrated interstitial macrophage and leukocyte infiltration. Furthermore, immunofluorescence analysis of six biopsies derived from the COVID-19 patients demonstrated direct correlation between the increased ACE2 levels and impaired spermatogenesis indicating possible mechanism of infection of testis by SARS-CoV-2 [29]. Since the expression of ACE2 is high in testes, $\mathrm{Li}$ et al. [30] concluded that SARS-CoV-2 enters the testicular interstitium via the circulation route during peak viremia and that Leydig cells could be one of the initial targets. Moreover, the testicular ACE2 expression has been related to age, the largest ACE2 expression was observed in 30 -years-old patients, while 60 -year-old patients showed the lowest expression in testes [31] suggesting that younger males with COVID-19 were at significantly higher risk for testicular damage compared to older males.

Efficient entry of SARS-CoV-2 into the host cells depends not only on the presence of ACE2 receptors but also on the transmembrane protease serine 2 (TMPRSS2), which cleaves S protein of the human coronaviruses on the membrane priming them for viral entry into the cells [32]. In addition to ACE2, TMPRSS2 is also expressed in spermatogonia, Leydig cells, and Sertoli cells, providing potential route of entry for SARS-CoV-2 into these cells [33]. The virus is expected to bind to ACE2 and TMPRSS2 in the testicular tissue to induce its adverse effects. Importantly, Abobaker and Raba [34] highlighted the possibility for testicular injury and subsequent male infertility after SARS-CoV-2 infection, which might be caused by either direct viral invasion through ACE2 receptors or indirectly via the inflammatory immune response.
SARS-CoV-2 induced inflammatory response. While cytokines play a vital role in the testicular function, they also participate in pathological processes [35]. Elevated concentrations of cytokines following viral infection can influence spermatogenesis and steroidogenesis, thereby seriously impacting fertility [36] (figure). Notably, testicular inflammation causes upregulation of interleukin (IL)-1 $\beta$, IL- $1 \alpha$, IL-6, and tumor necrosis factor- $\alpha$, which produce harmful effects on the germ cells and inflammatory conditions in testes that interfere with the process of spermatogenesis [37]. Moreover, ACE2 present on Leydig cells may also influence local microvascular flows and permeability and promote inflammation that interferes with the role of Leydig cells, thereby inhibiting testosterone production and damaging seminiferous tubular cells [5]. The SARS-CoV-2 could invade the male reproductive tract during acute infection through ACE2 present on the cells of seminiferous tubules. The virus could probably stay for only a few days due to the privileged immune status of testes [22]. In particular, the immunosuppressive characteristics of Sertoli cells and testicular macrophages are known to play a vital role in suppressing inflammation and reducing testicular damage associated with viruses. Nevertheless, the COVID-19associated inflammation may temporarily affect integrity of the blood-testis barrier (BTB), which could negatively impact spermatogenesis [33]. SARS-CoV-2 could cause an increase of ACE2 expression and promote typical inflammatory response that could interfere with the function of Leydig and Sertoli cells. Proinflammatory cytokines released by Leydig and Sertoli cells may activate the autoimmune response and damage the seminiferous epithelium, leading to autoimmune orchitis [5]. Accordingly, despite their privileged immune status, testes cannot be protected from the general immune response. Infiltration of leukocyte as well as $\mathrm{CD}^{3+} \mathrm{T}$ lymphocytes and $\mathrm{CD}^{68+}$ macrophages into the interstitial tissue of testis can generate interferons that may also decrease testosterone production [36]. Moreover, testosterone depletion has also been associated with autoimmune diseases and elevation of the inflammatory biomarkers such as C-reactive protein (CRP), IL-6, and TNF- $\alpha$ [38].

COVID-19-associated fever. The hyperactivated immune responses along with cytokine storms following SARS-CoV-2 infection affect many organs, including heart, liver, kidney, and testes. Interestingly, fever, is an additional risk of the COVID-19 that may affect male fertility. More importantly, hyperinflammatory condition with persistent fever, fulminant, and fatal hypercytokinemia have been associated with multi-organ failure [39]. High inflammatory response associated with fever, immune cell activation, and inflammatory mediators such as interferons and cytokines can affect testicular function [40, 41] (figure). The hypothesis that fever and elevation of testicular temperature contribute to sperm 


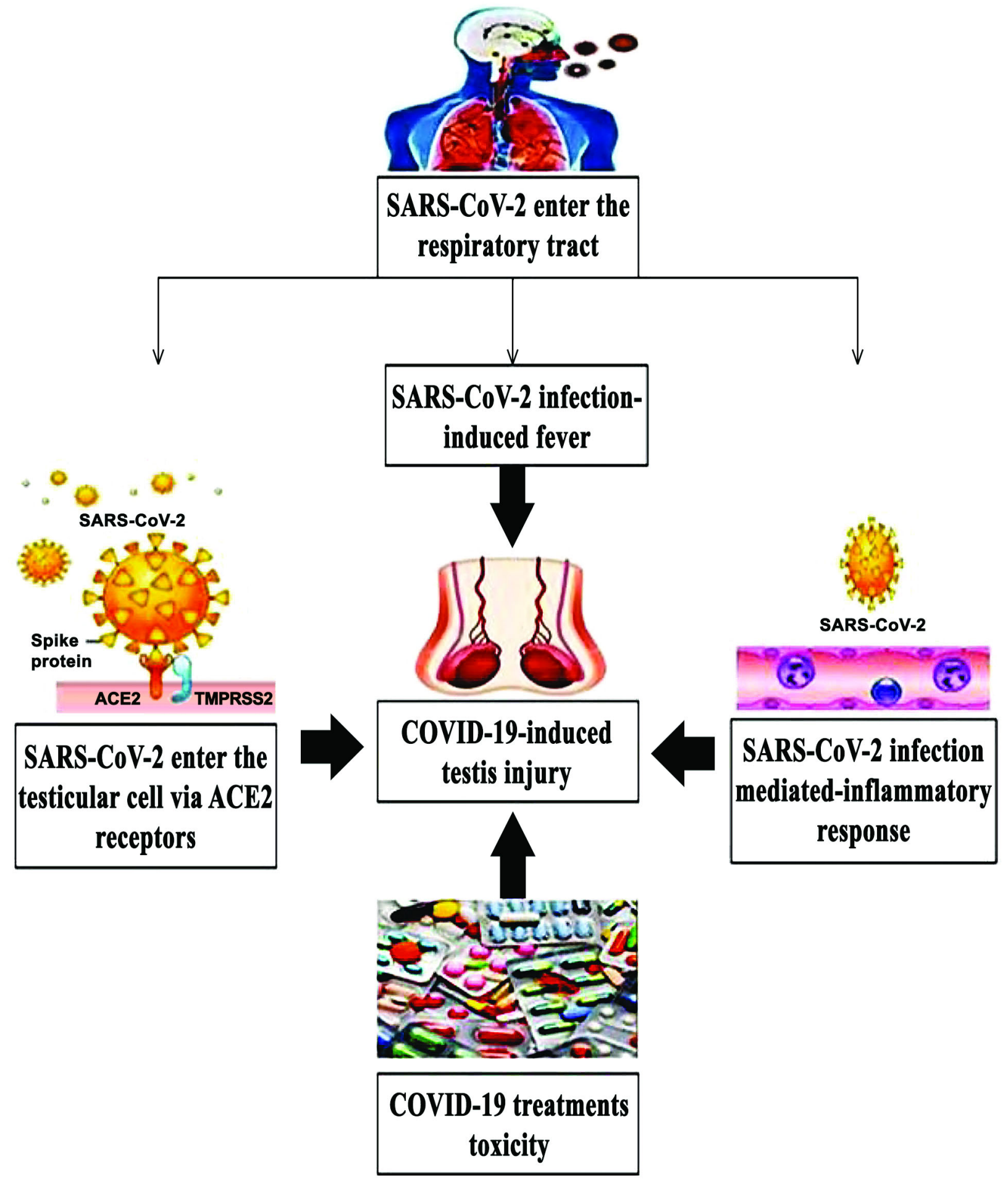

Summary of the probable pathogeneses of COVID-19-induced testicular injury. Designations: ACE2, angiotensin-converting enzyme 2; SARS-CoV-2, severe acute respiratory syndrome coronavirus 2. (Color version the figure is available in online version of the article and can be accessed at: https://www.springer.com/journal/10541)

deficiency has been generally accepted. Given that spermatogenesis could be affected by COVID-19-associated fever, semen parameters such as sperm concentration and motility could be decreased for 72 to 90 days after the viral infection [42]. Moreover, participants with verified COVID-19-associated fever tended to have lower motile sperm count, sperm concentration, and total sperm count [43]. 
COVID-19 and gonadal-toxic drugs. Interferon- $\alpha$ and ribavirin (in combination with interferon or lopinavir/ritonavir), as well as chloroquine phosphate, have been recommended for COVID-19 treatment [44]. Animal studies have revealed that ribavirin administration reduced testosterone levels and inhibited spermatogenesis [45], while lopinavir/ritonavir has also been observed to inhibit spermatogenesis in rats probably due to oxidative stress and inflammation [46]. Unfortunately, testicular dysfunction could be also caused by glucocorticoids and infectious diseases-related stress due to psychological crises induced by COVID-19 [22] (figure). Additionally, chloroquine phosphate has been found to affect spermatogenesis and epididymal function in male rats, which indicates that certain medication can affect testicular function in the male patients with COVID-19 [47] (table).

Unfortunately, the aforementioned primary studies on male fertility have some limitations related to sample size, research methodology, and disease course. Further comprehensive investigations at all levels are therefore required to improve the levels of evidence and understanding of the impact of SARS-CoV-2 on male reproduction and testicular health. Fortunately, many researchers have recently launched a highly promising multidimensional andrological research project in men called the PROTEGGIMI study (prospective multidimensional andrological translational research project) to develop international collaboration for the data registry in hormonal and genomic studies in hopes of filling the gaps in the current knowledge on association between SARSCoV-2 and male frailty [48]

\section{THE IMPACT OF LONG-TERM VIRAL INFECTION ON REPRODUCTIVE FUNCTION}

Given the novelty of the COVID-19 pandemic, longterm studies on its influence on male fertility have been unavailable. Nonetheless, several studies have addressed the effects of long-term viral infection on male reproductive function. Several viruses, such as human papillomaviruses, hepatitis B (HBV), hepatitis $\mathrm{C}$ viruses (HCV), human herpes viruses, influenza viruses, cytomegaloviruses, HIV, mumps virus, Zika viruses, and Ebola viruses have been known to cause orchitis and influence male fertility [49]. Interestingly, the study on 298 patients with mumps orchitis found that $24 \%$ of adults and $38 \%$ of adolescents exhibited seminal abnormalities for up to three years after recovery. At least $24 \%$ of adults and $38 \%$ of young individuals had abnormal ejaculation even three years after orchitis [50]. Ultrasonography of eight patients with mumps orchitis revealed atrophic testes with an oblong shape, heterogeneous low echogenicity, and decreased vascularity 40 to 230 days after the initial diagnosis [51. Histological characteristics of testis from 57 autopsied patients with chronic HIV, suggested a slightly lower degree of spermatogenesis as well as increased thickening of the cell membrane and interstitial fibrosis [52]. Moreover, the male patients with chronic HCV infection showed lower total serum testosterone, sperm count, and progressive sperm motility, and abnormal sperm morphology compared to the healthy controls [53]. Altered sperms morphology with decreased sperms motility, viability, and concentration were observed in the semen from patients with chronic HBV infection compared to the healthy individuals [54].

Notably, Ebola RNA may be found in seminal fluid for periods exceeding 13 months after infection [55]. Moreover, the study on virus persistence following the 2014-2016 Ebola virus outbreak had reported the presence of Ebola RNA in seminal fluid up to 565 days after infection [56]. Additionally, among a cohort of 135 male patients with Ebola who were followed-up from 2015 to 2017 , $8 \%$ reported erectile dysfunction, whereas $12 \%$ showed decreased libido [57]. Regarding the Zika virus, Counotte et al. [58] reported the presence of Zika RNA in semen for a median duration of 40 to 370 days. However, Avelino-Silva et al. [59] found normal levels of the serum sex hormone and absence of the Zika RNA in the semen samples from six patients 1-year post-Zika infection, although impaired motility was observed in three samples and low sperm count was reported in one sample.

\section{CONCLUSIONS}

The current review suggests that the recent coronavirus pandemic COVID-19 could have various effects on male reproductive health. Although, the data on the male reproductive system in patients with COVID-19 have been scarce with most relevant evidence coming from the small-scale studies without available long-term follow-up data. Taken together, several etiopathogenic hypotheses have been suggested implying that the SARS-CoV-2 infection might be responsible for impairment of testicular function. ACE2 is primarily expressed in spermatogonia and Leydig and Sertoli cells of the human testes, which may lead to a testicular dysfunction in patients infected with SARS-CoV-2. Moreover, testicular damage may be attributed to the inflammatory responses and fever-associated inflammation, as well as medications used in the severe cases. Thus, clinical and translational (short-/long-term) investigations in larger cohorts of currently infected subjects are needed to evaluate the impact of COVID-19 on human spermatogenesis, determine protective and curative approaches against testicular injuries, and establish clear conclusions.

Ethics declarations. The author declares no conflicts of interest. This article does not contain any studies with human participants or animals performed by the author. 


\section{REFERENCES}

1. Wu, Z., and McGoogan, J. M. (2020) Characteristics of and Important Lessons from the Coronavirus Disease 2019 (COVID-19) outbreak in China: summary of a report of 72 314 cases from the Chinese center for disease control and prevention, JAMA, 323, 1239-1242.

2. World Health Organization (2020) WHO Coronavirus Disease (COVID-19) Dashboard. Data last updated: 2121/3/12, URL: https://covid19.who.int.

3. Zhou, F., Yu, T., Du, R., Fan, G., Liu, Y., et al. (2020) Clinical course and risk factors for mortality of adult inpatients with COVID-19 in Wuhan, China: a retrospective cohort study, Lancet, 395, 1054-1062.

4. Wang, T., Du, Z., Zhu, F., Cao, Z., An, Y., et al. (2020) Comorbidities and multi-organ injuries in the treatment of COVID-19, Lancet, 395, e52, doi: 10.1016/S01406736(20)30558-4.

5. Xu, J., Qi, L., Chi, X., Yang, J., Wei, X., et al. (2006) Orchitis: a complication of severe acute respiratory syndrome (SARS), Biol. Reprod., 74, 410-416.

6. Corona, G., Baldi, E., Isidori, A. M., Paoli, D., Pallotti, F., et al. (2020) SARS-CoV-2 infection, male fertility and sperm cryopreservation: a position statement of the Italian Society of Andrology and Sexual Medicine (SIAMS) (Società Italiana di Andrologia e Medicina della Sessualità), J. Endocrinol. Invest., 43, 1153-1157.

7. Verdecchia, P., Cavallini, C., Spanevello, A., and Angeli, F. (2020) The pivotal link between ACE2 deficiency and SARS-CoV-2 infection, Eur. J. Intern. Med., 76, 14-20.

8. Li, R., Yin, T., Fang, F., Li, Q., Chen, J., et al. (2020) Potential risks of SARS-CoV-2 infection on reproductive health, Reprod. Biomed. Online, 41, 89-95.

9. Ding, Y., He, L., Zhang, Q., Huang, Z., Che, X., et al. (2004) Organ distribution of severe acute respiratory syndrome (SARS) associated coronavirus (SARS-CoV) in SARS patients: implications for pathogenesis and virus transmission pathways, J. Pathol., 203, 622-630.

10. Gagliardi, L., Bertacca, C., Centenari, C., Merusi, I., Parolo, E., et al. (2020) Orchiepididymitis in a Boy With COVID-19, Pediatr. Infect. Dis. J., 39, e200-e202.

11. Pan, F., Xiao, X., Guo, J., Song, Y., Li, H., et al. (2020) No evidence of severe acute respiratory syndrome-coronavirus 2 in semen of males recovering from coronavirus disease 2019, Fertil. Steril., 113, 1135-1139.

12. Nunes Duarte-Neto, A., de Almeida Monteiro, R. A., da Silva, L., Malheiros, D., de Oliveira, E. P., et al. (2020) Pulmonary and systemic involvement of COVID-19 assessed by ultrasound-guided minimally invasive autopsy, Histopathology, 77, 186-197, doi: 10.1111/his.14160.

13. Yang, M., Chen, S., Huang, B., Zhong, J. M., Su, H., et al. (2020) Pathological findings in the testes of COVID-19 patients: clinical implications, Eur. Urol. Focus, 6, 11241129.

14. Rastrelli, G., Di Stasi, V., Inglese, F., Beccaria, M., Garuti, M., et al. (2020) Low testosterone levels predict clinical adverse outcomes in SARS-CoV-2 pneumonia patients, Andrology, 9, 88-98, doi: 10.1111/andr.12821.

15. Çayan, S., Uğuz, M., Saylam, B., and Akbay, E. (2020) Effect of serum total testosterone and its relationship with other laboratory parameters on the prognosis of coronavirus disease 2019 (COVID-19) in SARS-CoV-2 infected male patients: a cohort study, Aging Male, doi: 10.1080/ 13685538.2020.1807930.

16. Ma, L., Xie, W., Li, D., Shi, L., Ye, G., et al. (2020) Evaluation of sex-related hormones and semen characteristics in reproductive-aged male COVID-19 patients, J. Med. Virol., doi: 10.1002/jmv.26259.

17. Schroeder, M., Tuku, B., Jarczak, D., Nierhaus, A., Bai, T., et al. (2020) The majority of male patients with COVID-19 present low testosterone levels on admission to intensive care in Hamburg, Germany: a retrospective cohort study, medRxiv, doi: 10.1101/2020.05.07.20073817.

18. Pal, R., and Banerjee, M. (2020) COVID-19 and the endocrine system: exploring the unexplored, J. Endocrinol. Invest., 43, 1027-1031.

19. Iglesias, P., Prado, F., Macías, M. C., Guerrero, M. T., Muñoz, A., et al. (2014) Hypogonadism in aged hospitalized male patients: prevalence and clinical outcome, J. Endocrinol. Invest., 37, 135-141.

20. Cardona Maya, W. D., Du Plessis, S. S., and Velilla, P. A. (2020) SARS-CoV-2 and the testis: similarity with other viruses and routes of infection, Reprod. Biomed. Online, $\mathbf{4 0 ,}$ 763-764.

21. Society for Assisted Reproductive Technology (SART) (2020) SART and ASRM issue advice for infertility patients concerning the novel coronavirus (COVID-19), URL: https://www.Sart.Org/news-and-publications/news-andresearch/press-releases-and-bulletins/sart-and-asrmissue-advice-for-infertility-patients-concerning-thenovel-coronavirus-covid-19/.

22. Li, D., Jin, M., Bao, P., Zhao, W., and Zhang, S. (2020) Clinical characteristics and results of Semen tests among men with Coronavirus disease 2019, JAMA Netw. Open, 3, e208292, doi: 10.1001/jamanetworkopen.2020.8292.

23. Song, C., Wang, Y., Li, W., Hu, B., Chen, G., et al. (2020) Absence of 2019 novel coronavirus in semen and testes of COVID-19 patients, Biol. Reprod., 103, 4-6, doi: 10.1093/ biolre/ioaa050.

24. Holtmann, N., Edimiris, P., Andree, M., Doehmen, C., Baston-Buest, D., et al. (2020) Assessment of SARS-CoV-2 in human semen - a cohort study, Fertil. Steril., 114, 233-238.

25. Paoli, D., Pallotti, F., Colangelo, S., Basilico, F., Mazzuti, L., et al. (2020) Study of SARS-CoV-2 in semen and urine samples of a volunteer with positive naso-pharyngeal swab, J. Endocrinol. Invest., 43, 1819-1822, doi: 10.1007/s40618-020-01261-1.

26. Ning, J., Li, W., Ruan, Y., Xia, Y., Wu, X., et al. (2020) Effects of 2019 Novel Coronavirus on male reproductive system: a retrospective study, Preprints, doi: 10.20944/ preprints202004.0280.v1.

27. Liu, X., Chen, Y., Tang, W., Zhang, L., Chen, W., et al. (2020) Single-cell transcriptome analysis of the novel coronavirus (SARS-CoV-2) associated gene ACE2 expression in normal and non-obstructive azoospermia (NOA) human male testes, Sci. China Life Sci., 63, 1006-1015.

28. Younis, J. S., Abassi, Z., and Skorecki, K. (2020) Is there an impact of the COVID-19 pandemic on male fertility? The ACE2 connection, Am. J. Physiol. Endocrinol. Metab., 318, E878-E880.

29. Achua, J. K., Chu, K. Y., Ibrahim, E., Khodamoradi, K., Delma, K. S., et al. (2020) Histopathology and ultrastructural findings of fatal COVID-19 infections on testis, World J. Mens Health, 38, e56, doi: 10.5534/wjmh.200170. 
30. Li, M. Y., Li, L., Zhang, Y., and Wang, X. S. (2020) Expression of the SARS-CoV-2 cell receptor gene ACE2 in a wide variety of human tissues, Infect. Dis. Poverty, 9, 45, doi: 10.1186/s40249-020-00662-X.

31. Shen, Q., Xiao, X., Aierken, A., Yue, W., Wu, X., et al. (2020) The ACE2 expression in Sertoli cells and germ cells may cause male reproductive disorder after SARS-CoV-2 infection, J. Cell. Mol. Med., 24, 9472-9477.

32. Hoffmann, M., Kleine-Weber, H., Schroeder, S., Krüger, N., Herrler, T., et al. (2020) SARS-CoV-2 cell entry depends on ACE2 and TMPRSS2 and is blocked by a clinically proven protease inhibitor, Cell, 181, 271-280.e8.

33. Wang, Z., and Xu, X. (2020) scRNA-seq profiling of human testes reveals the presence of the ACE2 receptor, a target for SARS-CoV-2 infection in spermatogonia, Leydig and Sertoli cells, Cells, 9, 920, doi: 10.3390/cells9040920.

34. Abobaker, A., and Raba, A. A. (2020) Does COVID-19 affect male fertility? World J. Urol., doi: 10.1007/s00345020-03208-w.

35. Loveland, K. L., Klein, B., Pueschl, D., Indumathy, S., Bergmann, M., et al. (2017) Cytokines in male fertility and reproductive pathologies: immunoregulation and beyond, Front. Endocrinol., 8, 307, doi: 10.3389/fendo.2017.00307.

36. Hedger, M. P., and Meinhardt, A. (2003) Cytokines and the immune-testicular axis, J. Reprod. Immunol., 58, 1-26.

37. Guazzone, V. A., Jacobo, P., Theas, M. S., and Lustig, L. (2009) Cytokines and chemokines in testicular inflammation: a brief review, Microsc. Res. Tech., 72, 620-628.

38. Tsilidis, K. K., Rohrmann, S., McGlynn, K. A., Nyante, S. J., Lopez, D. S., et al. (2013) Association between endogenous sex steroid hormones and inflammatory biomarkers in US men, Andrology, 1, 919-928.

39. Mehta, P., McAuley, D. F., Brown, M., Sanchez, E., Tattersall, R. S., et al. (2020) COVID-19: consider cytokine storm syndromes and immunosuppression, Lancet, 395, 1033-1034.

40. Satie, A. P., Mazaud-Guittot, S., Seif, I., Mahé, D., He, Z., et al. (2011) Excess type I interferon signaling in the mouse seminiferous tubules leads to germ cell loss and sterility, J. Biol. Chem., 286, 23280-23295.

41. Hedger, M. P. (2011) Immunophysiology and pathology of inflammation in the testis and epididymis, J. Androl., 32, 625-640.

42. Jung, A., and Schuppe, H. C. (2007) Influence of genital heat stress on semen quality in humans, Andrologia, 39, 203-215.

43. Patel, D. P., Guo, J., and Hotaling, J. M. (2020) The jury is still out: COVID-19 and male reproduction, Fertil. Steril., 114, 257-258.

44. Rismanbaf, A., and Zarei, S. (2020) Liver and kidney injuries in COVID-19 and Their effects on drug therapy, a letter to editor, Arch. Acad. Emerg. Med., 8, e17.

45. Almasry, S. M., Hassan, Z. A., Elsaed, W. M., and Elbastawisy, Y. M. (2017) Structural evaluation of the peritubular sheath of rat's testes after administration of ribavirin: A possible impact on the testicular function, Int. J. Immunopathol. Pharmacol., 30, 282-296.
46. Adaramoye, O. A., Akanni, O. O., Adewumi, O. M., and Owumi, S. E. (2015) Lopinavir/ritonavir, an antiretroviral drug, lowers sperm quality and induces testicular oxidative damage in rats, Tokai J. Exp. Clin. Med., 40, 51-57.

47. Asuquo, O. R., Igiri, A. O, Olawoyin, O. O., and Eyong, E. U. (2007) Correlation of histological and histometric changes in rats testes treated with chloroquine phosphate, Niger J. Physiol. Sci., 22, 135-139.

48. Salonia, A., Corona, G., Giwercman, A., Maggi, M., Minhas, S., et al. (2020) SARS-CoV-2, testosterone and frailty in males (PROTEGGIMI): a multidimensional research project, Andrology, 9, 19-22, doi: 10.1111/andr.12811.

49. Khalili, M. A., Leisegang, K., Majzoub, A., Finelli, R., Panner Selvam, M. K., et al. (2020) Male fertility and the COVID-19 pandemic: systematic review of the literature, World J. Mens Health, 38, 506-520.

50. Barták, V. (1973) Sperm count, morphology and motility after unilateral mumps orchitis, J. Reprod. Fertil., 32, 491494.

51. Choi, H. I., Yang, D. M., Kim, H. C., Kim, S. W., Jeong, H. S., et al. (2020) Testicular atrophy after mumps orchitis: ultrasonographic findings, Ultrasonography, 39, 266-271.

52. De Paepe, M. E., and Waxman, M. (1989) Testicular atrophy in AIDS: a study of 57 autopsy cases, Hum. Pathol., 20, 210-214.

53. Hofny, E. R., Ali, M. E., Taha, E. A., Nafeh, H. M., Sayed, D. S., et al. (2011) Semen and hormonal parameters in men with chronic hepatitis C infection, Fertil. Steril., 95, 25572559.

54. Lorusso, F., Palmisano, M., Chironna, M., Vacca, M., Masciandaro, P., et al. (2010) Impact of chronic viral diseases on semen parameters, Andrologia, 42, 121-126.

55. Sissoko, D., Duraffour, S., Kerber, R., Kolie, J. S., Beavogui, A. H., et al. (2017) Persistence and clearance of Ebola virus RNA from seminal fluid of Ebola virus disease survivors: a longitudinal analysis and modelling study, Lancet Glob. Health, 5, e80-e88.

56. Oka, M. J., Choi, M. J., Baller, A., White, S., Rogers, E., et al. (2016) Prevention of sexual transmission of Ebola in Liberia through a national semen testing and counselling programme for survivors: an analysis of Ebola virus RNA results and behavioural data, Lancet Glob. Health, 4, e736-743.

57. De St Maurice, A., Ervin, E., Orone, R., Choi, M., Dokubo, E. K., et al. (2018) Care of Ebola survivors and factors associated with clinical Sequelae-Monrovia, Liberia, Open Forum Infect. Dis., 5, ofy239, doi: $10.1093 /$ ofid/ofy239.

58. Counotte, M. J., Kim, C. R., Wang, J., Bernstein, K., Deal, C. D., et al. (2018) Sexual transmission of Zika virus and other flaviviruses: a living systematic review, PLoS Med., 15, e1002611, doi: 10.1371/journal.pmed.1002611.

59. Avelino-Silva, V. I., Alvarenga, C., Abreu, C., TozettoMendoza, T. R., Canto, C., et al. (2018) Potential effect of Zika virus infection on human male fertility? Rev. Inst. Med. Trop. Sao Paulo, 60, e64, doi: 10.1590/S16789946201860064. 